\title{
ARTICLE
}

\section{Uveal melanoma-associated survival in Scotland}

\author{
Aaron Jamison $\mathbb{D}^{1} \cdot$ Lesley A. Bhatti ${ }^{2} \cdot$ Manvi M. Sobti ${ }^{1} \cdot$ Vikas Chadha $^{1} \cdot$ Paul Cauchi $^{1} \cdot$ Ewan G. Kemp ${ }^{1}$
}

Received: 11 December 2018 / Revised: 19 May 2019 / Accepted: 14 June 2019 / Published online: 25 October 2019

(c) The Author(s), under exclusive licence to The Royal College of Ophthalmologists 2019

\section{Learning Objectives}

Upon completion of this activity, participants will be able to:

1. Describe recorded cause of death and long-term melanoma-specific survival of patients with UM, according to an analysis of SOOS data

2. Determine long-term cancer-specific and all-cause survival of patients with UM, according to an analysis of SOOS data

3. Identify clinical implications of long-term all-cause and cause-specific survival of patients with UM, according to an analysis of SOOS data

\section{Continuing Medical Education}

In support of improving patient care, this activity has been planned and implemented by Medscape, LLC and Springer Nature. Medscape, LLC is jointly accredited by the Accreditation Council for Continuing Medical Education (ACCME), the Accreditation Council for Pharmacy Education (ACPE), and the American Nurses Credentialing Center (ANCC), to provide continuing education for the healthcare team.

Medscape, LLC designates this Journal-based CME activity for a maximum of 1.0 AMA PRA Category 1 Credit(s) ${ }^{\mathrm{TM}}$. Physicians should claim only the credit commensurate with the extent of their participation in the activity.

\section{Credit Hours}

1.0

Release date: 25 October 2019

Expiration date: 25 October 2020

Post-test link: https://medscape.org/eye/posttest 918527

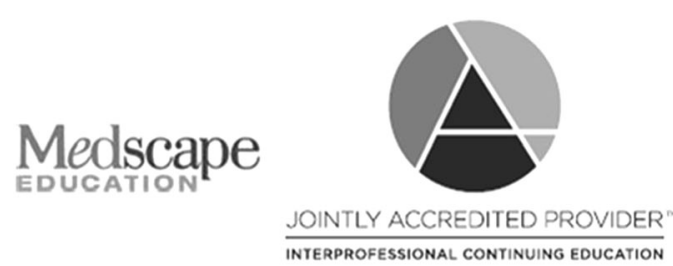

\section{Authors/Editors disclosure information}

S.S. has disclosed the following relevant financial relationships: Served as an advisor or consultant for: Allergan, Inc.; Bayer AG; Boehringer Ingelheim Pharmaceuticals, Inc.; Heidelberg Pharma GmbH; Optos; Roche. Served as a speaker or a member of a speakers bureau for: Allergan, Inc.; Bayer AG; Novartis Pharmaceuticals Corporation; Optos. Received grants for clinical research from: Allergan, Inc.; Bayer AG; Boehringer Ingelheim Pharmaceuticals, Inc.; Novartis Pharmaceuticals Corporation; Optos. A.J., L.B., M.S., V.C., P.C., and E.K. have disclosed no relevant financial relationships.

\section{Journal CME author disclosure information}

Laurie Barclay has disclosed no relevant financial relationships.

Aaron Jamison

Aaronjamison@gmail.com

1 Scottish Ocular Oncology Service, Tennent Institute of Ophthalmology, Glasgow, UK
2 Information Services Division, NHS National Services Scotland, Edinburgh, UK 


\section{Abstract}

Background/Objectives The Scottish Ocular Oncology Service (SOOS) manages all patients with uveal melanoma (UM) in Scotland. Our aim was to determine the long-term all-cause and cause-specific survival of patients with UM, irrespective of treatment modality.

Subjects/Methods A retrospective single-centre cohort study including all patients diagnosed with UM by the SOOS between 1/1/1998 and 31/12/2002. Data from the SOOS database were correlated with death records held by National Records of Scotland, which provided date, and all listed causes, of death for all deceased patients.

Results Two hundred and eighteen patients were newly diagnosed with UM between 1/1/1998 and 31/12/2002. One hundred and fifteen (52.8\%) were female. The mean (median) age at diagnosis was 63(65) years (range: 16-89). Of 179 choroidal melanomas, melanoma-specific survival was $92.3 \%, 87.4 \%$ and $83.8 \%$ at 5,10 and 15 years, respectively. Cancerspecific survival was $85.8 \%, 71.8 \%$ and $62.3 \%$. All-cause survival was $81.6 \%(146 / 179), 62.0 \%(111 / 179)$ and $46.7 \%$ (computed, fraction inexpressible). Of 26 ciliary body melanomas, melanoma-specific survival was $87.2 \%, 81.3 \%$ and $81.3 \%$ at 5,10 and 15 years. Cancer-specific survival was $62.9 \%, 40.6 \%$ and $40.6 \%$. All-cause survival was $61.5 \%$ (16/26), $38.5 \%(10 / 26)$ and $26.9 \%$ (7/26). Of 13 iris melanomas, at all three timepoints (5/10/15 years), melanoma-specific survival was $100 \%$, cancer-specific survival was $92.3 \%$, and all-cause survival was $76.9 \%(10 / 13)$.

Conclusions Correlation of SOOS and national records survival data confirms 15-year melanoma-specific survival of 83.8\%, $81.3 \%$ and $100 \%$ for choroidal, ciliary body and iris melanomas, respectively. We can now provide accurate survival data to our patients in Scotland.

\section{Introduction}

Uveal melanomas (UMs) are the most common form of primary intraocular malignancy and usually present as a pigmented mass within the choroid, ciliary body or iris. UM are rare, with an estimated incidence of six per million per year with no gender predilection, although they are 8-10x more common in Caucasian populations than nonCaucasian populations [1-3].

UM are often asymptomatic, although may present with varying degrees of visual loss. UM usually undergo haematogenous spread that can lead to hepatic metastatic disease and ultimately death, variably reported to occur in 23.3-50\% of cases within 15 years of diagnosis [4-12]. Treatment options range from localised globe-preserving options, such as brachytherapy, proton beam therapy (PBT), en bloc resection, endoresection and transpupillary thermotherapy, to enucleation, but none has been proven to be successful in improving survival [4-6, 13-16].

The development of hepatic metastasis has been associated with numerous risk factors including increasing tumour dimensions (thickness and/or diameter), involvement of the ciliary body, the presence of extraocular extension and increasing age [17, 18]. Other tumour features present that may indicate a poor prognosis on histopathological examination include epithelioid cell type, the presence of closed microvascular loops, high mitotic activity, the presence of tumour-infiltrating lymphocytes and tumour-infiltrating macrophages, and higher expression of insulin-like growth factor-1 receptor and human leukocyte antigen Class I and II. In addition, $1 \mathrm{p}$ loss, 6q loss, 8q gain and monosomy 3 all indicate a poorer prognosis [8].
In 2010, the American Joint Committee on Cancer (AJCC) released the 7th edition of the AJCC Cancer Staging Manual [19]. This was the first edition to include a staging system for UM (choroid and ciliary body), based on many of the risk factors mentioned in the preceding paragraph: largest basal tumour dimension, tumour thickness, and the presence of ciliary body involvement, extrascleral extension, lymph node involvement and/or distant metastasis. The AJCC staging system has become the most popular means by which ocular oncologists classify UM in order to estimate their patients' outcomes.

This study was performed by the Scottish Ocular Oncology Service (SOOS), which manages all patients with UM in Scotland. Our primary aim was to determine the long-term all-cause and cause-specific survival of patients with $\mathrm{UM}$, irrespective of their treatment modalities.

\section{Materials and methods}

A retrospective review of the SOOS patient database was performed, recruiting all patients diagnosed with UM (choroidal melanoma, ciliary body melanoma or iris melanoma) between 1st January 1998 and 31st December 2002. This provided patient identifiers, clinical details and survival status (including cause of death where relevant) for patients diagnosed with UM over this 5-year period.

Further review of the patient's clinical details was undertaken, utilising the patient's case notes and/or electronic clinical record (accessed via Clinical Portal, Orion Health), radiological imaging, PBT treatment reports (where available) and the histopathological reports of all enucleations performed. This process enabled cross-checking of the 
clinical details held by the SOOS patient database (in particular, the survival status \pm cause of death), and collection of data regarding tumour size, location, and the presence of extraocular extension, lymph node involvement or metastasis. The above recruitment time period was chosen to allow 15-year follow-up in our cohort, but the SOOS was not yet performing cytogenetic analysis on histopathological specimens at this time. Therefore, these data are not available for any of the patients in this cohort.

The above data were correlated with death records held by the National Records of Scotland, accessed via the Scottish Cancer Registry (Information Services Division [ISD] Scotland), which provided the date of death and all listed causes of death for all deceased patients within our cohort. Death was assumed in all cases where survival status differed between sources.

Survival analysis was performed using the Kaplan-Meier (KM) method for each tumour subtype (choroid, ciliary body and iris). Cause of death data was used to perform cause-specific survival analyses (cancer-specific and UMspecific, hereafter referred to as "melanoma-specific"). The classification of case diagnoses and causes of death was based on the International Statistical Classification of Diseases and Related Health Problems-ICD-9 (1979-1999) and ICD-10 (2000 onwards). Cancer-specific deaths were defined by the presence of at least one of: ICD-9 codes 140209 or 235-239; or ICD-10 codes B21, C00-C97 or D37D48. Melanoma-specific deaths were defined by the presence of at least one of: ICD-9 codes 190.5, 190.6, 190.8, 190.9 or 197.7 (liver metastasis); or ICD-10 codes C69.2, C69.3, C69.4, C69.9 or C78.7 (liver metastasis). The analysis separately searched for the presence of any of in situ neoplasms (ICD-9 codes 230-234, ICD-10 codes D00-D09) or benign neoplasms (ICD-9 codes 210-229, ICD-10 D10D36) as a failsafe, but none of these codes was identified.

It must be noted that ICD-9 did not offer specific codes for malignancies of the ciliary body or iris, and ICD-10, for those of the iris. However, the codes included in our analyses offer a wide enough scope to detect any eye malignancy, assuming that our patients with UM did not already possess or develop another, unrelated, eye malignancy. Of note, none of the ocular neoplasm ICD codes (ICD-9 codes starting 190, ICD-10 codes starting C69) that were not included in the definition of a "melanoma-specific death" were observed at any stage in the analysis.

Summary statistics for survival at 5, 10 and 15-year follow-up were highlighted. Kaplan-Meier graphs for each UM subtype were produced using the SPSS software. Survival was censored on the 31st December 2016 so that only patients diagnosed before 31 st December 2001 had at least 15-year follow-up. Our analysis assumed that all cases were diagnosed between the ages 15 and 99, which all cases were. Cause-specific survival also excluded deceased patients with missing underlying cause of death information, of which there were none. Unlike many other survival analyses that only include the underlying (primary) cause of death, all causes of death listed have been included in calculating causespecific survival.

The choroidal and ciliary body melanomas were categorized using the TNM classification system outlined in the AJCC Cancer Staging Manual, and subsequently staged, as described by the same system, into the categories I, IIA-B and IIIA-C [19, 20]. Kaplan-Meier survival analysis of each staging classification was performed.

The survival outcomes of our cohort were compared with a similarly-aged cohort of the Scottish general population. The details of this are outlined in the results section.

The NHS Health Research Authority confirmed that no ethical approval was required. This study adhered to the tenets of the Declaration of Helsinki.

\section{Results}

Two hundred and twenty patients were diagnosed with a UM by the SOOS between 1st January 1998 and 31st December 2002. Of these, two patients were identified as a recurrence of a previously diagnosed UM and were excluded from the analysis. Of the 218 remaining patients, 117 $(52.7 \%)$ were female and the mean (median) age at diagnosis was 63 (65) years with a range of 16-89 years old. Basic demographic data are presented in Table 1, alongside summarised data regarding tumour thickness and largest basal diameter.

One hundred and eighty-two of the 205 patients $(88.8 \%)$ with choroidal or ciliary body melanoma had sufficient data to enable AJCC tumour classification and staging. Tumour stages of these patients are presented in Table 2, accompanied by those reported in the initial study $(n=7369$, staging available for 5403) outlining this staging system, for comparison [20]. Ten-year melanoma-specific survival rates from both studies are presented.

\section{Recorded cause of death}

Of the 179 patients with choroidal melanoma, 98 died within the study period. Of these 98 deaths, the death certificate reported liver metastasis or UM as a cause of death in 24 cases (24.5\%), and cancer (of any form) was listed as a cause of death in 63 cases $(64.3 \%)$.

Of the 26 patients with ciliary body melanoma, 19 died within the study period. Of these, the death certificate reported liver metastasis or UM as a cause of death in four cases $(21.1 \%$ ), and cancer (of any form) was listed as a cause of death in 14 cases $(73.7 \%)$. 
Table 1 Patient demographics and tumour dimensions by uveal melanoma type

\begin{tabular}{|c|c|c|c|c|}
\hline & 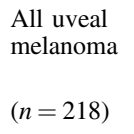 & $\begin{array}{c}\begin{array}{c}\text { Choroidal } \\
\text { melanoma }\end{array} \\
(n=179)\end{array}$ & $\begin{array}{l}\text { Ciliary } \\
\text { body } \\
\text { melanoma } \\
(n=26)\end{array}$ & $\begin{array}{l}\begin{array}{l}\text { Iris } \\
\text { melanoma }\end{array} \\
(n=13)\end{array}$ \\
\hline \multicolumn{5}{|l|}{ Age (years) } \\
\hline Mean & 63 & 63 & 60 & 61 \\
\hline Median & 65 & 65 & 63 & 71 \\
\hline Range & $16-89$ & $29-89$ & $16-82$ & $23-83$ \\
\hline \multicolumn{5}{|l|}{ Gender $(n,(\%))$} \\
\hline Male & $103(47.2)$ & $86(48.0)$ & $11(42.3)$ & $6(46.2)$ \\
\hline Female & $115(52.8)$ & $93(52.0)$ & $15(57.7)$ & $7(53.8)$ \\
\hline \multicolumn{5}{|c|}{ Tumour dimensions (mm) } \\
\hline $\begin{array}{l}\text { Availability of } \\
\text { information } \\
(n,(\%))\end{array}$ & - & $\begin{array}{l}156 / 179 \\
(87.1 \%)\end{array}$ & $\begin{array}{l}23 / 26 \\
(88.5 \%)\end{array}$ & $\begin{array}{l}\text { Not } \\
\text { measured }\end{array}$ \\
\hline $\begin{array}{l}\text { Largest basal } \\
\text { diameter (mean) }\end{array}$ & - & 11.6 & 14.5 & N/A \\
\hline $\begin{array}{l}\text { Largest basal } \\
\text { diameter (range) }\end{array}$ & - & $2.7-21.2$ & $4.5-25.0$ & N/A \\
\hline $\begin{array}{l}\text { Tumour } \\
\text { thickness (mean) }\end{array}$ & - & 6.1 & 9.2 & N/A \\
\hline $\begin{array}{l}\text { Tumour thickness } \\
\text { (range) }\end{array}$ & - & $1.0-18.0$ & $2.0-16.0$ & $N / A$ \\
\hline
\end{tabular}

Table 2 Patient cohort (ciliary body and choroidal melanoma only) by American Joint Committee on Cancer (AJCC) tumour stage, compared with Kujala et al. ${ }^{\mathrm{a}}$

\begin{tabular}{|c|c|c|c|c|c|c|}
\hline \multirow[t]{2}{*}{ Stage } & \multicolumn{3}{|c|}{ Current study $(n=182 / 205)$} & \multicolumn{3}{|c|}{ Kujala et al. (JCO) $(n=5403 / 7369)$} \\
\hline & $n$ & $\%$ & $\begin{array}{l}\text { 10-year melanoma- } \\
\text { specific survival }\end{array}$ & $n$ & $\%$ & $\begin{array}{l}\text { 10-year melanoma- } \\
\text { specific survival }\end{array}$ \\
\hline I & 49 & 26.9 & $97.7 \%$ & 1119 & 20.7 & $88 \%$ \\
\hline IIA & 55 & 30.2 & $94.4 \%$ & 1705 & 31.6 & $80 \%$ \\
\hline IIB & 39 & 21.4 & $77.9 \%$ & 1217 & 22.5 & $67 \%$ \\
\hline IIIA & 29 & 15.9 & $75.0 \%$ & 913 & 16.9 & $45 \%$ \\
\hline IIIB & 7 & 3.8 & Too few cases & 383 & 7.1 & $27 \%$ \\
\hline IIIC & 3 & 1.6 & Too few cases & 66 & 1.2 & Too few cases \\
\hline
\end{tabular}

Number of patients $(n)$, percentage distribution $(\%)$ and 10-year melanoma-specific survival rates $(\%)$ for comparative purposes

${ }^{a}$ The original study that led to development of the updated ciliary body and choroidal melanoma classification and staging system outlined in the seventh edition of the Cancer Staging Manual of the American Joint Committee on Cancer

Of the 13 patients with iris melanoma, three died within the study period. Of these, the death certificate reported liver metastasis or UM as a cause of death in none of the cases. Cancer (of any form) was listed as a cause of death in one case $(33.3 \%)$.

\section{Melanoma-specific survival}

For choroidal melanoma, melanoma-specific survival was $92.3 \%, 87.4 \%$ and $83.8 \%$ at 5,10 and 15 years, respectively. For ciliary body melanoma, melanomaspecific survival was $87.2 \%$ at 5 years, and $81.3 \%$ at both 10 and 15 years. And for iris melanoma, melanoma-specific survival was the same, $100 \%$, at 5, 10 and 15 years. Kaplan-Meier survival results are presented in Fig. 1, while Table 3 displays the survival estimates.

\section{Cancer-specific survival}

For choroidal melanoma, cancer-specific survival was $85.8 \%, 71.8 \%, 62.3 \%$ at 5,10 and 15 years, respectively. For ciliary body melanoma, cancer-specific survival was $62.9 \%, 40.6 \%$ and $40.6 \%$ at 5, 10 and 15 years. And for iris melanoma, cancer-specific survival was the same, $92.3 \%$, at 5, 10 and 15 years.

\section{All-cause survival}

For choroidal melanoma, all-cause survival was $81.6 \%$ (146/179), 62.0\% (111/179) and 46.7\% (fraction cannot be expressed as survival rate was computed due to the presence of censored cases), at 5, 10 and 15 years, respectively. For ciliary body melanoma, all-cause survival was $61.5 \%$ (16/ 26), 38.5\% (10/16) and 26.9\% (7/26) at 5, 10 and 15 years. And for iris melanoma, all-cause survival was the same, $76.9 \%(10 / 13)$, at 5, 10 and 15 years.

Survival estimates for all of these findings can be found in Table 3. The large 95\% confidence intervals seen with the results for ciliary body and iris melanoma mortality reflect the limited accuracy of these results due to relatively small numbers of cases (and deaths) analysed.

Finally, in collaboration with the National Records of Scotland, we identified a cohort of the Scottish general population that, similar to a proportion of our study population, were aged 65 in $1998(n=48,599)$ [21]. This age also matches the median age of our cohort. Mid-year population estimates (which are matched to the results of each UK census) were used to quantify the number of people in this original cohort who had died by the years 2003, 2008 and 2013. This allowed the calculation of 5, 10 and 15-year all-cause survival rates for the general population (aged 65 in 1998) that were 90.3\%, 77.5\% and $61.4 \%$, respectively. Cancer-related deaths in this same cohort (as provided by the Scottish Cancer Registry) were used to further calculate the cancer-specific "mortality rates" of the general population (aged 65 in 1998) that were $4.0 \%, 8.9 \%$ and $14.3 \%$ for 5,10 and 15 years, respectively.

\section{Discussion}

This study has been performed in collaboration with the Scottish Cancer Registry which, operated by ISD Scotland, 
aims to deliver timely, comparable and high-quality cancer data [22]. Its data are self-audited by a variety of quality assurance systems, and is externally audited by the United Kingdom and Ireland Association of Cancer Registries (UKIACR).

The Scottish Cancer Registry retrieves data regarding death from National Records of Scotland (NRS), who in turn retrieve this information from the Medical Certificate of Cause of Death (MCCD). In the case of data regarding cause of death, the listed diagnoses are converted to ICD-9 (1979-1999) and ICD-10 (2000 onwards) codes outlined by the International Statistical Classification of Diseases and Related Health Problems. NRS are regulated by the UK Statistics Authority-an independent body directly accountable to Parliament, responsible for upholding the quality and comprehensiveness of official statistics.

Correlation of survival data from the SOOS database and national records confirms 15 -year UM-specific survival of $83.8 \%, 81.3 \%$ and $100 \%$ for choroidal, ciliary body and iris melanomas, respectively. Amongst the existing literature, Kujala et al. [7] reported metastatic melanoma-related survival in patients with either choroidal or ciliary body melanoma of $50 \%$ at 15 years, McLean et al. [10] reported mortality from metastasis in $46 \%$ at 15 years, while Frenkel et al. [4] reported a 15-year melanoma-related mortality rate of $23.3 \%[4,7,10]$. Our results suggest that the survival in patients with UM is greater in our population.

We believe that the disparity of our findings with those previously published may be due in part to the high quality of the data obtained from NRS through the Scottish Cancer Registry, and the privilege of the SOOS in caring for all patients with UM in Scotland-our study population. The Scottish population may differ from those reported in the literature in terms of ethnicity and genetic factors. In additon, the Scottish General Ophthalmic Services (GOS) contract provides regular free eye examinations to all, with almost universal availability of retinal cameras, enabling the early detection of smaller UMs [23]. This may be reflected by the larger proportion of smaller tumours seen in our cohort when compared with those reported by Kujala et al., as outlined in Table 2 [20]. Early detection of small tumours may be associated with reduced risk of metastasis, and enables earlier treatment, also possibly associated with reduced risk of metastasis [8, 14]. Finally, many of the seminal publications that detailed UM-associated prognosis included only patients who had undergone enucleation, at a time when smaller tumours were observed, or possibly managed less invasively $[7,10,20]$. The inclusion of smaller tumours, diagnosed on clinical grounds, distinguishes our cohort (and others similar) from those that included only patients that had undergone enucleation.

Considering our patients with ciliary body melanoma, although the observed melanoma-specific survival rate was the
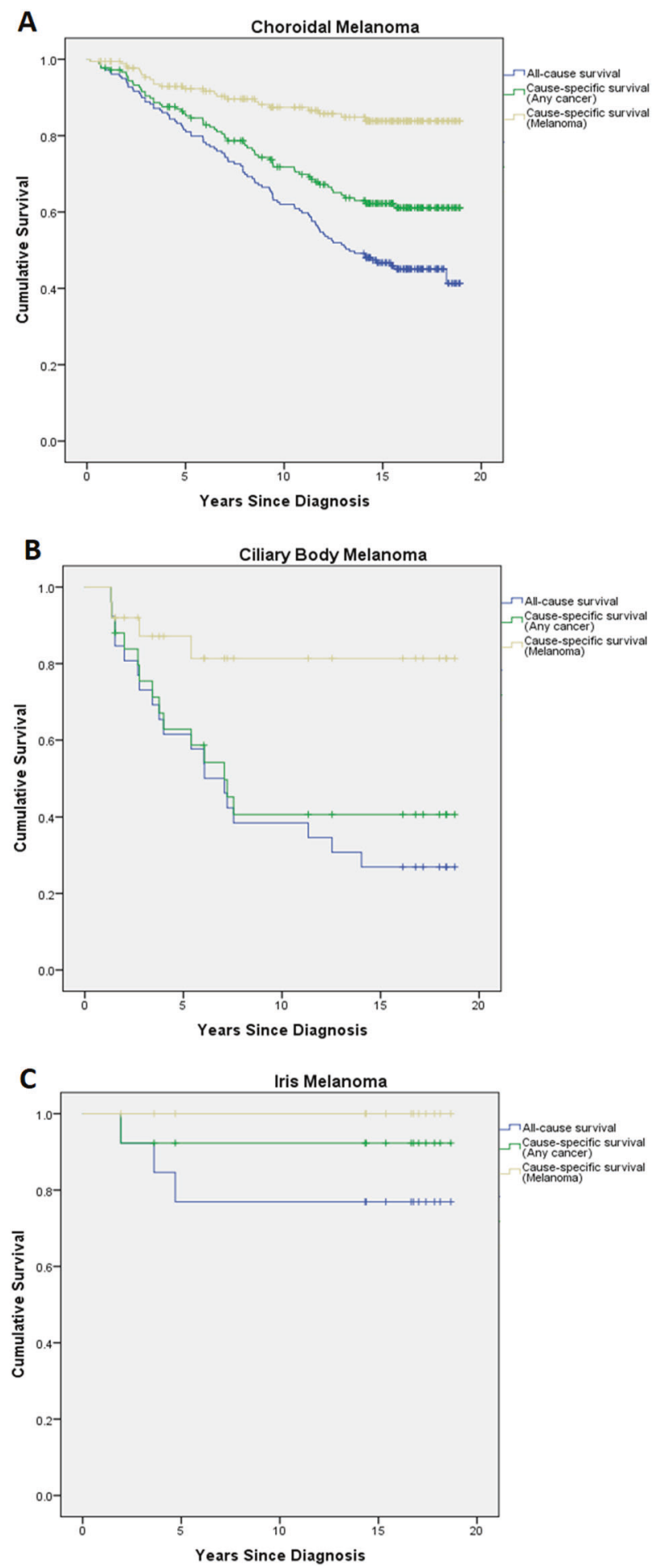

Fig. 1 Kaplan-Meier survival plots-a choroidal melanoma, b ciliary body melanoma and $\mathbf{c}$ iris melanoma. All-cause survival (bottom line), cancer-specific survival (middle line) and melanoma-specific survival (top line) are plotted

lowest of the three cohorts, all of these deaths had occurred within three years of diagnosis. As such, ciliary body melanoma was seen to cause a larger number of deaths in a shorter 
Table 3 Summary of survival at 5,10 and 15 years (\%) with corresponding $95 \%$ confidence intervals

\begin{tabular}{|c|c|c|c|c|c|c|}
\hline & \multicolumn{2}{|c|}{ Choroidal melanoma } & \multicolumn{2}{|c|}{$\begin{array}{l}\text { Ciliary body } \\
\text { melanoma }\end{array}$} & \multicolumn{2}{|c|}{ Iris melanoma } \\
\hline & \multicolumn{2}{|l|}{$(n=179)$} & \multicolumn{2}{|l|}{$(n=26)$} & \multicolumn{2}{|l|}{$(n=13)$} \\
\hline & Survival & $95 \% \mathrm{CI}$ & Survival & $95 \% \mathrm{CI}$ & Survival & $95 \% \mathrm{CI}$ \\
\hline \multicolumn{7}{|l|}{5 years } \\
\hline Melanoma-specific survival & 92.3 & $87.1-95.4$ & 87.2 & $65.0-95.7$ & 100 & N/A \\
\hline Cancer-specific survival & 85.8 & $79.7-90.2$ & 62.9 & $40.7-78.7$ & 92.3 & $56.6-98.9$ \\
\hline All-cause survival & 81.6 & $75.1-86.5$ & 61.5 & $40.3-77.1$ & 76.9 & $44.2-91.9$ \\
\hline \multicolumn{7}{|l|}{10 years } \\
\hline Melanoma-specific survival & 87.4 & $81.1-91.7$ & 81.3 & $57.0-92.7$ & 100 & N/A \\
\hline Cancer-specific survival & 71.8 & $64.4-78.0$ & 40.6 & $21.0-59.4$ & 92.3 & $56.6-98.9$ \\
\hline All-cause survival & 62.0 & $54.5-68.7$ & 38.5 & $20.4-56.3$ & 76.9 & $44.2-91.9$ \\
\hline \multicolumn{7}{|l|}{15 years } \\
\hline Melanoma-specific survival & 83.8 & $76.7-89.0$ & 81.3 & $57.0-92.7$ & 100 & N/A \\
\hline Cancer-specific survival & 62.3 & $54.3-69.3$ & 40.6 & $21.0-59.4$ & 92.3 & 56.6-98.9 \\
\hline All-cause survival & 46.7 & $39.2-53.8$ & 26.9 & $11.9-44.5$ & 76.9 & $44.2-91.9$ \\
\hline
\end{tabular}

period of time than choroidal or iris melanoma, with limited associated mortality after this initial high-risk period.

Using data from the National Records of Scotland, we calculated 5, 10 and 15-year all-cause survival rates for the general population (aged 65 in 1998) of 90.3\%, 77.5\% and $61.4 \%$, respectively. Separately, melanoma-specific "mortality rates" for the choroidal melanoma group were calculated by subtracting the calculated survival fractions $(0.923$, 0.874 and 0.838 at 5,10 and 15 years) from 1 . These "mortality rates" $(7.7 \%, 12.6 \%, 16.2 \%$ at 5,10 and 15 years) were combined with the same group's all-cause survival rates $(81.6 \%, 62.0 \%$ and $46.7 \%$, respectively) to identify the survival that might be hypothetically expected if that patient did not have a UM $(89.3 \%, 74.6 \%$ and $62.9 \%$ at 5,10 and 15 years). These survival rates correspond closely with the all-cause survival rates for the general population calculated above $(90.3 \%, 77.5 \%$ and $61.4 \%$, respectively). Although it is not practical to compare these groups directly, we believe that the strength of our results is supported by the close similarities of the general population's calculated all-cause survival rates to the choroidal melanoma group's all-cause survival rates plus the same cohort's calculated melanoma-specific "mortality rates".

In our analyses, the difference between the cancer-specific survival rate and the melanoma-specific survival rate represents the percentage of patients that died of a second cancer. For the choroidal melanoma group, this percentage is $6.5 \%$, $15.6 \%$ and $21.5 \%$ for 5,10 and 15 years. These percentages are higher at each timepoint than cancer-specific survival rates of the general population $(4.0 \%, 8.9 \%$ and $14.3 \%$, respectively) calculated in collaboration with the Scottish Cancer Registry. It is unclear what proportion of this disparity is due to a possibly increased rate of second cancers in Scotland, and what is due to melanoma-related deaths that have been missed by our analysis.

Of the patients reported to be alive by the NRS, none were identified as deceased on correlation with the SOOS database or the patient's case notes and/or electronic clinical record (accessed via Clinical Portal, NHS Scotland). This suggests that the identification of deceased patients by the NRS is accurate.

Possible inaccuracy of the underlying/primary cause of death and poor completion of the MCCD by the reporting clinician may affect the accuracy of data obtained from the MCCD [24, 25]. We hope to have compensated for the possibility of having missed deaths due to UM by the inclusion of cancer-specific and all-cause survival data.

Interrogation of the SOOS patient database, and subsequently the patients' clinical records, enabled the collection of the reported dataset. It did not provide complete data on the treatments that patients received for UM. The wide range of available local UM treatments has been discussed, although none of these has been clearly proven to have any effect on patient survival [14, 26, 27]. As such, our survival analysis has been performed without considering tumour treatments received.

The SOOS serves the population of Scotland $(5,424,800$ in 2017) and benefits from being able to report the characteristics of UM in a country with a stable population [21]. Our findings will be useful in counselling patients with new and previous diagnoses of UM within our service.

A further, larger, study performed in a population comparable to Scotland's would be of clinical value to ascertain whether there is geographical variation in UM prognosis, or possibly, whether the overall prognosis of UM is improving. A recently published study by Baily et al. reports another discrete 
stable population - the neighbouring Republic of Irelandwhich describes a similar epidemiology of UM, since the establishment of their dedicated ocular oncology service in 2010 [28]. Survival rates have been calculated to four years (88\% overall cancer-specific survival, 95\% CI:82-93) and longer follow-up will allow comparison of survival outcomes with this centre, and hopefully others, in the near future.

Correlation of survival data from the SOOS database and national records confirms 15-year melanoma-specific survival of $83.8 \%, 81.3 \%$ and $100 \%$ for choroidal, ciliary body and iris melanomas, respectively. These results contrast with frequently cited findings of $50 \%$ metastatic melanomarelated survival at 15 -years. The authors hope that these higher survival rates are due in part to the earlier detection and treatment of smaller UMs in Scotland, but acknowledge that our results are limited by a smaller cohort size, and by the potential under-reporting of melanoma metastases.

\section{Summary}

\section{What was known before}

- Uveal melanoma (UM) usually undergoes haematogenous spread that can lead to hepatic metastatic disease and ultimately death, variably reported to occur in $23.3-50 \%$ of cases within 15 years of diagnosis.

- The Scottish Ocular Oncology Service (SOOS) manages all patients with UM in Scotland.

\section{What this study adds}

- Correlation of Scottish Ocular Oncology Service and national records survival data confirms 15-year melanoma-specific survival of $83.8 \%, 81.3 \%$ and $100 \%$ for choroidal, ciliary body and iris melanomas, respectively.

- We can now provide relevant survival data to our patients in Scotland.

Acknowledgements Jaroslaw Lang (Information Services Division, NHS National Services Scotland, Meridian Court, Glasgow, Scotland) for assistance with statistical analysis. Susan Ewan (Administrator, Scottish Ocular Oncology Service, Tennent Institute of Ophthalmology, Glasgow, UK) for continued support and management of the SOOS, including maintenance of the SOOS database, and assistance with data acquisition.

\section{Compliance with ethical standards}

Conflict of interest The authors declare that they have no conflict of interest.

Publisher's note Springer Nature remains neutral with regard to jurisdictional claims in published maps and institutional affiliations.

\section{References}

1. Singh AD, Bergman L, Seregard S. Uveal melanoma: epidemiologic aspects. Ophthalmol Clin North Am. 2005;18:75-84.

2. Shields CL, Kaliki S, Cohen MN, Shields PW, Furuta M, Shields JA. Prognosis of uveal melanoma based on race in 8100 patients: the 2015 Doyne Lecture. Eye. 2015;29:1027-35.

3. McLaughlin CC, Wu XC, Jemal A, Martin HJ, Roche LM, Chen VW. Incidence of noncutaneous melanomas in the U.S. Cancer. 2005;103:1000-7.

4. Frenkel S, Hendler K, Pe'er J. Uveal melanoma in Israel in the last two decades: characterization, treatment and prognosis. Isr Med Assoc J. 2009;11:280-5.

5. Singh AD, Turell ME, Topham AK. Uveal melanoma: trends in incidence, treatment, and survival. Ophthalmology. 2011; 118:1881-5.

6. Virgili G, Gatta G, Ciccolallo L, Capocaccia R, Biggeri A, Crocetti E. et al. EUROCARE working group, survival in patients with uveal melanoma in Europe. Arch Ophthalmol. 2008; 126:1413-8.

7. Kujala E, Mäkitie T, Kivelä T. Very long-term prognosis of patients with malignant uveal melanoma. Invest Ophthalmol Vis Sci. 2003;44:4651-9.

8. Kaliki S, Shields CL, Shields JA. Uveal melanoma: estimating prognosis. Indian J Ophthalmol. 2015;63:93-102.

9. The Collaborative Ocular Melanoma Study Group. Development of metastatic disease after enrolment in the COMS trials for treatment of choroidal melanoma. COMS report no. 26. Arch Ophthalmol. 2005;123:1639-43.

10. McLean IW, Foster WD, Zimmerman LE. Uveal melanoma: location, size, cell type, and enucleation as risk factors in metastasis. Hum Pathol. 1982;13:123-32.

11. Seregard S, Kock E. Prognostic indicators following enucleation for posterior uveal melanoma. A multivariate analysis of longterm survival with minimized loss to follow-up. Acta Ophthalmol Scand. 1995;73:340-4.

12. Seddon JM, Albert DM, Lavin PT, Robinson N. A prognostic factor study of disease-free interval and survival following enucleation for uveal melanoma. Arch Ophthalmol. 1983;101: 1894-9.

13. Jovanovic P, Mihajlovic M, Djordjevic-Jocic J, Vlajkovic S, Cekic S, Stefanovic V. Ocular melanoma: an overview of the current status. Int J Clin Exp Pathol. 2013;6:1230-44.

14. Damato B. Does ocular treatment of uveal melanoma influence survival? Br J Cancer. 2010;103:285-90.

15. Singh AD, Topham A. Survival rates with uveal melanoma in the United States: 1973-1997. Ophthalmology. 2003;110:962-5.

16. The Collaborative Ocular Melanoma Study Group. The Collaborative Ocular Melanoma Study (COMS) randomized trial of pre-enucleation radiation of large choroidal melanoma IV: tenyear mortality findings and prognostic factors. COMS report no. 24. Am J Ophthalmol. 2004;138:936-51.

17. Shields CL, Furuta M, Thangappan A. Metastasis of uveal melanoma millimeter-by-millimeter in 8033 consecutive eyes. Arch Ophthalmol. 2009;127:989-98.

18. Shields CL, Kaliki S, Furuta M, Mashayekhi A, Shields JA. Clinical spectrum and prognosis of uveal melanoma based on age at presentation in 8,033 cases. Retina. 2012;32:1363-72.

19. Edge SB, Byrd DR, Compton CC (eds). AJCC Cancer Staging Manual, 7th edn. Springer: New York, NY; 2010.

20. Kujala E, Damato B, Coupland SE, Desjardins L, Bechrakis NE, Grange JD. et al. Staging of ciliary body and choroidal melanomas based on anatomic extent. J Clin Oncol. 2013;31:2825-31.

21. National Records of Scotland, Edinburgh, UK. http://www. nrscotland.gov.uk/. Accessed 15 Apr 18. 
22. Information Services Division Scotland, National Services Scotland, NHS Scotland, UK. http://www.isdscotland.org/. Accessed 15 Apr 18

23. Scottish Government. Part I - context and developments since 2006. In: Community eyecare services review. Scotland, UK: Scottish Government. https://www.gov.scot/Publications/2017/04/ 7983. Accessed 30 Aug 18.

24. Roulson J, Benbow EW, Hasleton PS. Discrepancies between clinical and autopsy diagnosis and the value of post mortem histology; a meta-analysis and review. Histopathology. 2005;47:551-9.
25. Maudsley G, Williams EM. Death certification by house officers and general practitioners-practice and performance. J Public Health Med. 1993;15:192-201.

26. Damato B. Progress in the management of patients with uveal melanoma. The 2012 Ashton Lecture. Eye. 2012;26:1157-72.

27. Damato B. Legacy of the collaborative ocular melanoma study. Arch Ophthalmol. 2007;125:966-8.

28. Baily C, O’Neill V, Dunne M, Cunningham M, Gullo G, Kennedy $\mathrm{S}$, et al. Uveal melanoma in Ireland. Ocul Oncol Pathol. 2019;5:195-204. 